\title{
Methods of Determining the Desired Frequency Characteristics of the Automatic Control Systems
}

\author{
A.K. Ablesimov \\ National Aviation University \\ Aviation Computer- \\ Integrated Complexes \\ Department \\ Kyiv, Ukraine \\ alexander.ablesimov@gmail.com
}

\author{
M.A. Pylypenko \\ National Aviation University \\ Aviation Computer- \\ Integrated Complexes \\ Department \\ Kyiv, Ukraine
}

\author{
L.V. Pogribnyak \\ National Aviation University \\ Aviation Computer- \\ Integrated Complexes \\ Department \\ Kyiv, Ukraine \\ ludmila_pogrebnjak@ukr.net
}

\author{
N.V. Usenko \\ National Aviation University \\ Aviation Computer- \\ Integrated Complexes \\ Department \\ Kyiv, Ukraine
}

\begin{abstract}
Considered the methods of determining the desired frequency characteristics of the automatic control systems while the synthesis of their corrective devices.

Keywords - correction device; inverse transfer function; spectral density; amplitude and phase frequency characteristic; the desired frequency characteristics; inverse amplitude and phase frequency characteristic
\end{abstract}

\section{INTRODUCTION}

The generalized structural scheme of the automatic control system is shown at the Fig. 1.

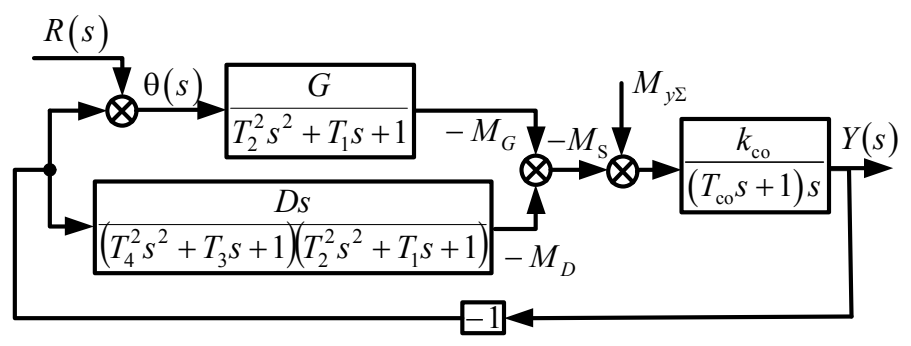

Fig. 1. The structural scheme of the automatic control system.

The illustrated structural scheme does not always allow to obtain the required quality of the control processes at the selected design parameters of elements. Besides feedback by the absolute angular velocity of the control object into the structure of the regulator of the stabilization system are usually introduced an additional parallel and serial corrective devices to ensure a given quality.

Parallel corrective devices are implemented as rigid and flexible feedbacks covering an element, a chain of elements or the entire system. Hard negative feedbacks are used relatively rarely for the correction of quality. This is because that the introduction of negative rigid feedback, providing speed increasing and expansion of the linearity zone, at the same time reduces the transfer coefficient of the covered element or the chain of elements. As a result - increasing of the control errors. The most widely for the purposes of quality correction are applied the flexible feedbacks - feedbacks from the derived output value of the element (a chain of elements) or system. Since the flexible feedbacks are acting only during the transients, their introduction allows to correct the quality of governance without changing the static properties of the system.

Serial corrective devices are usually realized in the form of differentiating or integro-differentiating passive DC circuits. The differentiating circuits along with the expansion of the area of stability of the system improve its action in time, providing the forcing of transients.

Raising the margin of stability of the system while simultaneously forcing of the transients - important advantage of the successive differentiating contours. The widespread use of these contours for the correction the quality of system is also caused by the simplicity of their constructive realization.

Much less often differentiating circuits apply the integrating SCC. Application of the integrating circuit (ideal) allows to increase an order of magnitude system astatism, but leads to deterioration of its stability.

\section{Problem Statement}

In order to choose one or the other type of corrective device and determine its parameters, you must compare the original (uncorrected) system with the desired system. Comparisons are usually conducted on logarithmic amplitude and phase frequency characteristics.

The main difficulty of choosing of corrective devices for the automatic control systems is to determine of the species of desired frequency characteristics to provide the desired quality of control. This explained primarily by the fact that in the technical conditions for the design are usually set requirements 
that characterize the efficiency of the system as a whole, rather than separate indicators of quality. Consequently the desired characteristics is often necessary to build approximately according to very general requirements for the quality of the control.

Below we consider two cases for determining the desired characteristics, that most frequently meet in the synthesis of corrective devices of automatic control systems.

\section{DETERMINATION OF THE CHARACTERISTICS}

A. Determination of the desired frequency characteristics of the ACS by a given type of transition

While designing the ACS can be assigned not the individual parameters of the control quality, but characteristic of the transition process, which occurs in the system under the action of the control signal or external perturbation. Required characteristic of the transition process can be tentatively constructed also with the help of its individual parameters $t_{\mathrm{rt}}, t_{\mathrm{ps}}, y_{\mathrm{osh}}, n$.

Usually given the characteristic of the transition process as the reaction on a step change of the control signal or the external disturbance under zero initial conditions (Fig. 2a). This kind of characteristic is not satisfy the conditions of absolute integrability and can not be represented by a Fourier integral.

With this in mind, let us consider the function

$$
y(t)=Y(t)-Y_{\text {st }},
$$

satisfying the conditions of absolute integrability, since when $t \rightarrow \infty \quad y(t) \rightarrow 0$ (Fig. 2b).

Applying to (1) direct Fourier transformation, we obtain

$$
\int_{0}^{\infty} y(t) e^{-j \omega t} d t=\frac{W(j \omega)}{j \omega}-\frac{Y_{\mathrm{st}}}{j \omega} .
$$

After transformation the equation (2), we obtain the amplitude and phase frequency characteristic of a closed system:

$$
W(j \omega)=Y_{\mathrm{st}}+j \omega \int_{0}^{\infty} y(t) e^{-j \omega t} d t
$$

The real and imaginary parts of the APFC are respectively:

$$
\left.\begin{array}{c}
P(\omega)=Y_{\mathrm{st}}+\omega \int_{0}^{\infty} y(t) \sin \omega t d t \\
Q(\omega)=\omega \int_{0}^{\infty} y(t) \cos \omega t d t .
\end{array}\right\}
$$

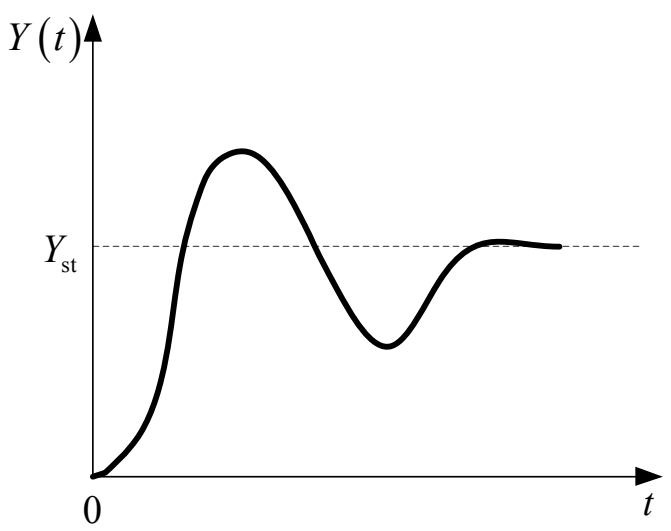

a

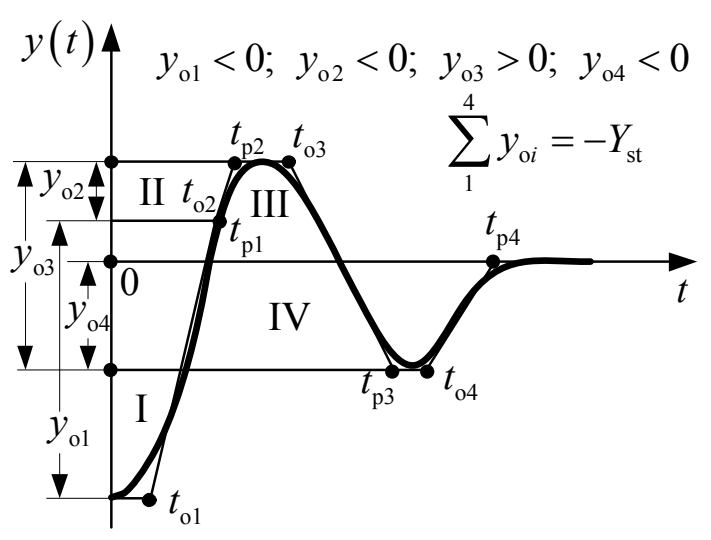

b

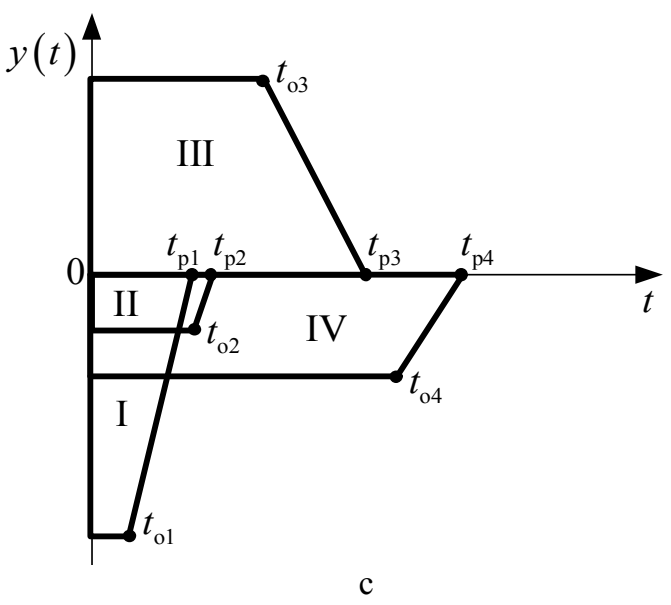

Fig. 2. Approximation of the transition process by trapezoids: (a) is the initial $Y(t)$; (b) is characteristic $y(t)=Y(t)-Y_{\text {st }} ;($ c) is trapezes.

The integrals in (4) can be accurately calculated only for the simplest functions $y(t)$. Therefore it is advisable to use for the approximate calculations $P(\omega)$ and $Q(\omega)$ the trapezoidal components of the function $y(t)$ instead of the function itself. 
If $y(t)$ can be approximately represented as a rectangular trapezoid with bases $t_{\mathrm{p}}$ and $t_{\mathrm{o}}$ and height $y_{\mathrm{o}}$, the integrals will be equal:

$$
\begin{aligned}
& \omega \int_{0}^{\infty} y(t) \sin \omega t d t=y_{\mathrm{o}}+\frac{y_{\mathrm{o}}}{\omega} \cdot \frac{\sin t_{\mathrm{p}} \omega-\sin t_{\mathrm{o}} \omega}{t_{\mathrm{p}}-t_{\mathrm{o}}} ; \\
& \omega \int_{0}^{\infty} y(t) \cos \omega t d t=\frac{y_{\mathrm{o}}}{\omega t_{\mathrm{p}}} \cdot \frac{\cos t_{\mathrm{o}} \omega-\cos t_{\mathrm{p}} \omega}{t_{\mathrm{p}}-t_{\mathrm{o}}} .
\end{aligned}
$$

When approximating the characteristic $y(t)$ by multiple trapezoids (Fig. 2c) so that $\sum_{1}^{i} y_{\mathrm{oi}}=-Y_{\mathrm{st}}$, we obtain:

$$
\begin{aligned}
& P(\omega)=Y_{\mathrm{st}}+\sum_{1}^{i} P_{i}(\omega)=\frac{1}{\omega} \sum_{1}^{i} y_{\mathrm{o} i} \frac{\sin t_{\mathrm{pi}}\left(\omega-\sin t_{\mathrm{o} i} \omega\right.}{t_{\mathrm{pi}}-t_{\mathrm{o} i}} ; \\
& Q(\omega)=\sum_{1}^{i} Q_{i}(\omega)=\frac{1}{\omega} \sum_{1}^{i} y_{\mathrm{oi}} \frac{\cos t_{\mathrm{o} i} \omega-\cos t_{\mathrm{p} i} \omega}{\left(t_{\mathrm{pi}}-t_{\mathrm{o} i}\right) t_{\mathrm{p} i}} .
\end{aligned}
$$

By equation (6) is approximately calculated desired frequency characteristics of the system, a comparison of which with the frequency characteristics of the original (uncorrected) system allows you to choose the necessary correction devices for obtaining a predetermined transient.

$B$. Determination of the desired frequency characteristics of the $A C S$ by minimum of the output error

If we know the spectral density of a random external perturbation acting on the control object, the desired frequency response of the ACS should be determined by minimizing the output error

Let us assume that the spectral density $S_{x y}(\omega)$ of the total perturbation moment is known, for example, calculated on the basis of the spectral densities of the fluctuations of the movable base. Also known stability region of the ACS (calculated or derived experimentally). Inside this area is allocated the area of desired adjustments.

By choosing in the region of possible adjustments a number of points $(1,2,3,4)$ with different values $G$ and $D$, we build for each of them (Fig. 3) the reverse amplitude-phase frequency characteristics of the open-loop system

$$
E_{\mathrm{ol}}(j \omega)=U_{E}(\omega)+j V_{E}(\omega)
$$

and their corresponding inverse amplitude and phase frequency characteristics

$$
E(j \omega)=1+E_{\mathrm{ol}}(j \omega)=P_{E}(\omega)+j Q_{E}(\omega)
$$

of the closed system relative to the disturbance.
Then, for each of the selected operating points determine the spectral density of the output error of ACS

$$
S_{y}(\omega)=S_{s y}(\omega) A_{m y}^{2}(\omega)=\frac{S_{s y}(\omega)}{P_{E}^{2}(\omega)+Q_{E}^{2}(\omega)},
$$

where $A_{m y}(\omega)$ is the amplitude-frequency response of a closed system relative to the disturbance.

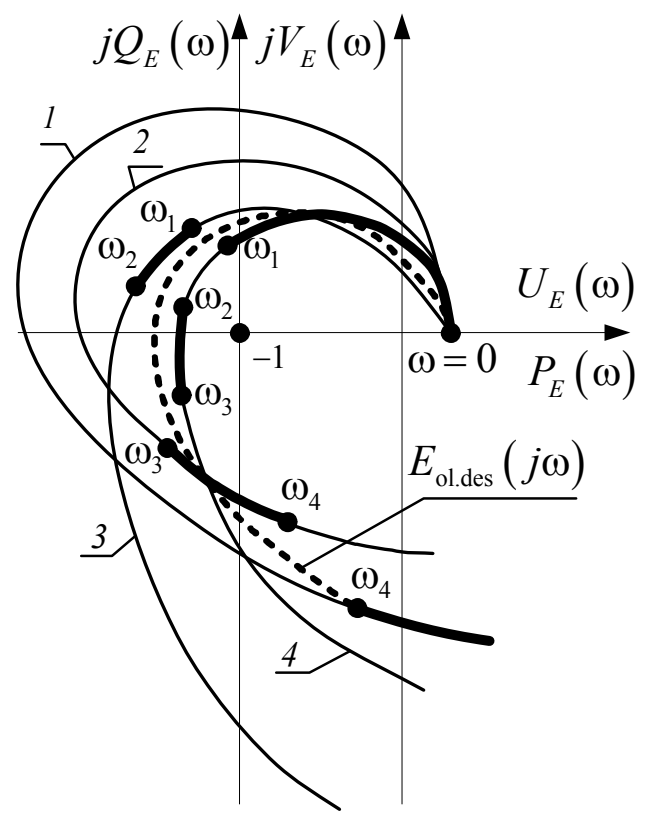

Fig. 3. Inverse APFC of the open-loop and closed-loop systems.

The founded spectral densities $S_{y}(\omega)$ are drawn on a single graph (Fig. 4). Graphically define the lower envelope (fatty bottom curve) of spectral densities errors.

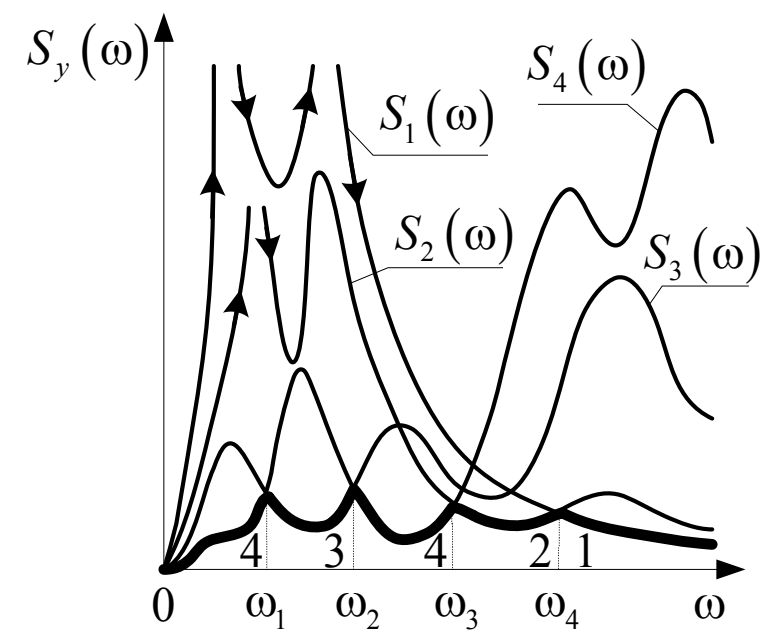

Fig. 4. The spectral densities of system errors. 
Frequency intervals $0-\omega_{1} ; \omega_{1}-\omega_{2} ; \omega_{2}-\omega_{3}$ and so on belong to the finite portions of different spectral densities of output error. They determine which of the baseline characteristics $E_{\mathrm{ol}}(j \omega)$ in this frequency range provides a minimum error of ACS. These portions are shown in characteristics of see Fig. 3.

Connecting them by a smooth line (dotted line in Fig. 3) is approximately getting the reverse amplitude-phase frequency characteristic of the desired system $E_{\text {oldes }}(j \omega)$. Knowing $E_{\text {ol.des }}(j \omega)$ we define $L_{\text {ol.des }}(j \omega)$ and by the usual method we choose the type parameters of the serial correcting device.

If the spectral density of the external disturbance is unknown, the intervals of frequencies, in which the desired characteristic $E_{\text {oldes }}(j \omega)$ coincides with the areas of initial characteristics $E_{\mathrm{ol}}(j \omega)$, can be approximately defined by the lower envelope of the family of the amplitude-frequency characteristics $A_{m y}(\omega)$ of the closed system.

In all cases, after an approximate determination of the desired frequency characteristics is advisable to check their compliance with the lower envelope of the $S_{y}(\omega)$ family or $A_{m y}(\omega)$, and if it is necessary, to clarify the approximating areas.

Let us note that the described method of determining the corrective devices can use as an input the experimental or the computational characteristics.

\section{CONCLUSIONS}

The main difficulty during the synthesis of automatic control systems (ACS) is the definition of the form of their desired frequency characteristics.

Knowing the last provides to get a frequency characteristics of correction circuits of ACS, thus ensuring the possibility of their constructive realization.

This article discusses two approaches to the definition of the desired frequency characteristics of ACS, which can be implemented during synthesis of their corrective devices:

- by the given type of the transition process;

- by the minimum of the stabilization error.

The considered methods for determining the desired characteristics allow to solve the problem of correcting the quality of ACS.

\section{REFERENCES}

[1] A.K. Ablesimov and L.V. Bardon, "Stability borders and regions of stabilization systems of inertial control objects". Electronics and Control Systems. Kyiv, NAU. 2014. no.4(42). pp. 35-38.

[2] A.K. Ablesimov and N.I. Kutova, "Evaluation of the quality of stabilization systems by normalized indirect indicators". Electronics and Control Systems. Kyiv, NAU. 2015. no.1(43). pp. 26-30.

[3] A.K. Ablesimov, L.V. Bardon, and N.I. Kutova, "Reaction of stabilization system on the commands of operator. Accuracy of stabilization". Electronics and Control Systems. Kyiv, NAU. 2016. no.1(47). pp. 16-21. 